\title{
A percepção sobre o acesso avançado em uma unidade unidade-escola de atenção básica à saúde
}

Perception of advanced access in a basic health care unit

\author{
Larissa Silva Soares ${ }^{1}$ (1) | larissamed70@gmail.com \\ Marcelle Aparecida de Barros Junqueira' (D) marcellebarros@ufu.br
}

\section{RESUMO}

Introdução: Uma das diretrizes da atenção básica à saúde (ABS) no Brasil é o primeiro contato da pessoa no sistema de saúde. O acesso ou a acessibilidade é um atributo essencial da APS que teve avaliação ruim em pesquisas brasileiras. O sistema de agendamento certamente influencia o acesso nas unidades de saúde, e o modelo por acesso avançado (AA) tem sido implantado no Brasil, e vem obtendo melhores resultados que modelos tradicionais de agendamento.

Objetivo: Este estudo teve como objetivo compreender a percepção de profissionais que atuam em uma unidade básica de saúde de Uberlândia, em Minas Gerais - MG (Centro de Saúde Escola Jaraguá (Cejar)), sobre a implementação do AA.

Método: Trata-se de um estudo qualitativo que utilizou o grupo focal e a análise de conteúdo.

Resultado: Foram delimitadas as seguintes categoriais temáticas: Desafios desafios na consolidação da APS e investimento em equipes mínimas; Tensionamentos tensionamentos quanto ao aumento na demanda de atendimentos individuais e sobrecarga de trabalho; Educação educação e saúde: intersecções necessárias entre ensino, serviço, gestão e comunidade.

Conclusão: O Cejar apresentou particularidades com a implementação do AA por ser uma unidade de ensino, por não ter Estratégia de Saúde da Família (ESF) consolidada e pela sobrecarga de trabalho advinda com dessa agenda, o que pode contribuir para o planejamento de outras equipes que tenham interesse pelo $A A$.

Palavras-chave: Atenção Básica à Saúde; Acesso aos serviços Serviços de saúdeSaúde; Percepção; Profissionais; Agendamento de Consultas.

\section{ABSTRACT}

Introduction: One of the guidelines of Primary Health Care (PHC) in Brazil is the person's user's first contact with the health system. Access or accessibility is an essential attribute of PHC that has been poorly assessed in Brazilian surveys. The appointment scheduling system certainly influences access in health units, and the model for advanced access (AA) has been implemented in Brazil, and has been achievinggetting better results than traditional scheduling models.

Objective: The objective of the presentthis study is to understand the perception of professionals who work in a basic health unit in Uberlândia-MG (Centro de Saúde Escola Jaraguá) about the implementation of $A$.

Method: It is a qualitative study that used the focus group methodology and content analysis.

Result: The following thematic categories were delimited: Challenges in the consolidation of PHC and investment in minimal teams; Tensions regarding the increasedin the demand for individual careassistance and work overload; Education and health: necessary intersections between teaching, service, management and community.

Conclusion: It was concluded that CEJAR presented specific characteristicsparticularities with the implementation of AA as it isfor being a teaching unit, doesfor not haveing a consolidated Family Health Strategy (FHS) and due to thefor the work overload resulting from this scheduleagenda, which can contribute to the planning of other teams that are interested in $A A$.

Keywords: Primary Health Care; Access to health services; Perception; Professionals; Appointment scheduling.

1 Universidade Federal de Uberlândia, Uberlândia, Minas Gerais, Brasil.

Editora-chefe: Rosiane Viana Zuza Diniz.

Editora associada: Rosiane Viana Zuza Diniz.

Recebido em 11/06/21; Aceito em 27/11/21.

Avaliado pelo processo de double blind review. 


\section{INTRODUÇÃO}

O acesso é condição primordial para que a atenção primária à saúde (APS) (também conhecida por "atenção básica à saúde" (ABS), no Brasil) garanta seus outros atributos de orientação familiar e comunitária, coordenação do cuidado, longitudinalidade e integralidade. Vários são os aspectos para oferecer acesso ao usuário: profissionais e infraestrutura não bastam se o indivíduo não consegue deles usufruir. O horário de funcionamento do serviço e os meios para se chegar até ele (transporte, barreiras geográficas, distância) precisam ser adequados ao perfil do usuário. Até a comunicação e os fatores culturais influenciam no acesso ${ }^{1,2}$. O acesso avançado (AA) diz respeito apenas à marcação de consultas médicas, ou seja, traz à tona apenas um dos diversos aspectos relacionados ao acesso do usuário.

A Estratégia Saúde da Família (ESF) tem como diretriz a garantia do acesso, no entanto normas claras e objetivas para o acesso oportuno nunca foram definidas em âmbito nacional ou local ${ }^{2,3}$. Estudos brasileiros sobre acesso e acolhimento foram publicados mostrando que a ESF ampliou o acesso aos serviços de saúde ${ }^{4-6}$. No entanto, fica claro que esse ainda é um problema de saúde do Brasil ${ }^{7}$, porque, mesmo com a cobertura por ESF já ampliada em algumas localidades ${ }^{8-10}$, as pesquisas com metodologia qualitativa demonstram dificuldade no acesso, como demora para conseguir marcar serviços (de uma a várias semanas) ${ }^{7,9,11-13}$. Já um estudo que utilizou a metodologia quantitativa mostrou as mesmas dificuldades de acesso ${ }^{14}$.

Assim, o acesso ainda é considerado um desafio na APS brasileira. Um dos motivos para isso seria a quantidade excessiva de pessoas vinculadas a uma única equipe de atenção básica ${ }^{15}$, que idealmente seria de duas mil a 3.500 pessoas $^{16}$. Outro motivo seria a centralidade do cuidado no médico ${ }^{17}$, e não nas demais categorias profissionais da equipe. O sistema de agendamento das unidades de APS também pode significar um entrave ao acesso do usuário, pela demora na marcação dos serviços ${ }^{7,12,13}$.

O tipo de agendamento de consultas pode aumentar o acesso à $\mathrm{APS}^{18}$. Se efetivo, aumenta a satisfação de profissionais e usuários, melhorando o ambiente de trabalho, a qualidade do cuidado e a segurança do paciente ${ }^{19}$. No sistema do agendamento por AA, não é feita distinção entre demanda programada e demanda espontânea. Os usuários são atendidos no mesmo dia em que chegam ao serviço, se assim o quiserem, independentemente do motivo da consulta. O lema é fazer o trabalho do dia no mesmo dia20,21, pois a maioria das vagas está reservada para demanda espontânea (de 65\% a 90\%) ${ }^{22}$.

Em tese, isso evitaria filas, pois o serviço estaria apto a absorver a demanda de usuários do dia, desde que o número da população adscrita por médico seja adequado ${ }^{23}$. Além disso, o AA em si não considera a lógica da equidade (as consultas são marcadas independentemente do motivo), mas o acolhimento, que é uma ferramenta das unidades de APS e de humanização do Sistema Único de Saúde (SUS) ${ }^{24}$, auxilia para que as consultas sejam organizadas por prioridades, classificações e/ ou complexidades. O AA permite que o usuário seja consultado no dia ou em até 48 horas, podendo agendar para depois se assim preferir ${ }^{21}$. Não há diferença entre consultas programadas e demanda espontânea ${ }^{23}$, dando-se ênfase à horizontalidade das ações em saúde ${ }^{21}$. Desde 2014, o AA vem sendo implantado em algumas unidades de APS, como iniciativas locais, para melhor atender à demanda populacional ${ }^{25}$. O estudo catarinense recente já citado demonstrou o efeito positivo do AA na qualidade dos cuidados ofertados em unidades de APS, por se tratar de um modelo de agendamento mais adequado às necessidades de saúde das pessoas ${ }^{22}$.

O acesso à APS é ainda motivo de insatisfação entre os usuários, principalmente pela demora na marcação e espera por consultas médicas. O sistema de agendamento por AA vem contribuindo para a diminuição da demora por consultas, à medida que "o trabalho de hoje é feito hoje". A revisão de literatura não apontou pontos negativos do $A A$. $A$ percepção de indivíduos que já vivenciam o AA contribuirá para o conhecimento sobre o tema no Brasil e poderá ter implicações em futuras ações de saúde relacionadas ao sistema de agendamento na APS.

Ademais, a formação de profissionais médicos e demais profissionais da área da saúde, desde a graduação, perpassando pela residência, até o doutoramento, requer a experiência in loco no cenário da APS, em toda a sua potência e suas fragilidades, sendo o acolhimento um espaço de potência para formação de competências clínicas, políticas e humanísticas. A imersão dos atores formais e informais do campo do "ensino" nos serviços da Rede de Atenção à Saúde é algo extremamente complexo. A transversalidade na formação-intervenção requer que o estudante vivencie simultaneamente os desafios da APS ao mesmo tempo que precisa "exercer" habilidades de escuta e acolhimento, e a prática de modelos organizativos dos serviços de saúde, incluindo a questão do acesso ${ }^{26,27}$.

No Centro de Saúde Escola Jaraguá (Cejar), unidade de $A B S$ da Universidade Federal de Uberlândia (UFU), o AA foi adotado de dezembro de 2018, com o intuito de diminuir a espera por consultas médicas programadas (que variava, na época, em torno de 45 dias) e a consequente insatisfação do usuário. Assim, com esta pesquisa, objetivouse compreender a percepção dos profissionais quanto à implantação do AA no Cejar. 


\section{MÉTODOS}

\section{Tipo de estudo}

Trata-se de um estudo de abordagem qualitativa, exploratório e baseado em grupo focal.

\section{Cenário de estudo}

Este estudo foi realizado no município de Uberlândia, no interior de Minas Gerais, que tem uma população estimada de 700 mil pessoas. O cenário do estudo foi o Cejar fundado em 1982 como uma das unidades didáticas avançadas (UDA) da Faculdade de Medicina (Famed) da UFU, em um convênio com a prefeitura de Uberlândia e a Fundação de Assistência, Estudo e Pesquisa de Uberlândia (Faepu). As UDA eram configuradas como cenários de prática para a graduação. $\mathrm{O}$ Cejar iniciou seu funcionamento como um ambulatório, no formato de uma unidade básica de saúde (UBS) tradicional (com clínico geral, pediatra, ginecologista e obstetra). Em 1999, criou-se o Centro de Referência Macrorregional no Serviço de Dermatologia Sanitária e Hansenologia do Hospital de Clínicas (HC/UFU), que funcionou no Cejar até 2018, em parceria com a UBS. Em 2012, a residência multiprofissional do HC/UFU é inserida no Cejar. De 2014 a 2016, uma unidade de saúde da família funcionou no Cejar, com uma médica cubana vinda do Programa Mais Médicos pelo Brasil.

Em 2018, a residência de medicina de família e comunidade (MFC) também é inserida no serviço, com a contratação de duas médicas de família e comunidade para a implementação de um novo modelo de gestão. Quando a residência de MFC entra no Cejar, o Serviço de Dermatologia Sanitária e Hansenologia sai, e ainda permanece o funcionamento da UBS tradicional. Dessa maneira, hoje o Cejar ainda é uma unidade de APS, que está em processo de implementação da ESF (ainda não conta com agentes comunitários de saúde - ACS), que recebe graduandos de Medicina, Enfermagem e Nutrição, além de residentes multiprofissionais e de MFC.

O Cejar está localizado no bairro Jaraguá, na cidade de Uberlândia. Sua área de abrangência é referente a todo o bairro Jaraguá e a uma parte do bairro Tubalina. De acordo com os dados de 2010 da prefeitura de Uberlândia ${ }^{28}$, todo o bairro Tubalina abrange uma população de cerca de 8.960 habitantes. Já o bairro Jaraguá tem uma população de 8.083 habitantes. A maioria da população está na faixa etária adulta e idosa. O perfil socioeconômico é considerado de classe média a classe média baixa. $O$ atual painel populacional é desconhecido, mas estima-se que seja de quatro mil pessoas, visto que recadastramentos feitos em 2018 e 2019 por alunos geraram dados pouco confiáveis.

\section{Participantes do estudo}

Os participantes, convidados mediante apresentação de Termo de Consentimento Livre e Esclarecido (TCLE), foram funcionários do Cejar: duas auxiliares administrativas, duas enfermeiras, uma médica assistente e preceptora da residência de MFC, além de três residentes do segundo ano de MFC da UFU. Eles foram escolhidos por terem vivido com proximidade o período de implementação do $\mathrm{AA}$, ou seja, esse era o critério de inclusão, e, assim, a amostra era intencional. As autoras do artigo não participaram do grupo, a fim de evitar possíveis interferências no debate; afinal, uma das autoras é médica de família e comunidade no Cejar.

\section{Coleta de dados}

As informações foram coletadas mediante a realização de um grupo focal (GF); essa técnica permite que os pesquisadores investiguem opiniões, valores, atitudes, crenças e influências grupais, de modo a gerar hipóteses, instrumentos e teorias. $\mathrm{O}$ ator principal é o grupo, e não individualidades. O GF faz com que a temática seja mais problematizada em relação a entrevistas individuais, criando reflexões coletivas e críticas quanto à implantação de programas na APS ${ }^{29}$.

O GF foi utilizado para coletar dados a partir da discussão e interação em grupo acerca do $\mathrm{AA}$, estimulado pelas seguintes questões norteadoras:

1. O que você conhecia sobre o AA antes de sua implementação no Cejar?

2. Como está sendo o processo de implementação do AA no Cejar?

3. Quais mudanças vocês percebem nos processos de trabalho da unidade com o AA?

4. Quais os aspectos positivos o AA trouxe para os processos de trabalho do Cejar? E quais os aspectos negativos?

5. Quais sugestões você daria para melhorar o funcionamento do AA no Cejar?

A duração do GF foi de cerca de 90 minutos, marcado com antecedência e com a autorização da chefia imediata. Os profissionais participaram do grupo durante sua jornada de trabalho em horário de reunião de equipe, no segundo semestre de 2020, no final do turno da manhã. O áudio foi gravado, e realizaram-se a observação e a relatoria de pontos considerados importantes. O grupo em círculo auxiliou a interação. Havia uma moderadora e uma relatora, colaboradoras da pesquisa, previamente capacitadas e com experiência em GF. A transcrição do áudio foi realizada pela mesma relatora do grupo, sendo as falas dos participantes indicadas por números aleatórios, preservando o anonimato deles. A conferência dos 
dados transcritos com os coletados (integridade dos dados) foi feita pelas autoras do artigo que não participaram do grupo.

\section{Análise de dados}

Os dados foram analisados mediante a técnica de análise de conteúdo proposta por Bardin ${ }^{30}$, a qual se configura como "um conjunto de técnicas de análise das comunicações, que utiliza procedimentos sistemáticos e objetivos de descrição do conteúdo das mensagens"(p.15), ocorrendo tanto análise dos conteúdos nas figuras de linguagem, reticências e entrelinhas, quanto dos manifestos ${ }^{31}$.

Produzir inferências sobre o texto objetivo é a razão de ser da análise de conteúdo ${ }^{32}$, a qual conta com várias etapas que serão brevemente descritas e foram sistematicamente realizadas nesta pesquisa: pré-exploração do material ou leitura flutuante (contato com os documentos e contexto, gerando impressões e orientações) ${ }^{30}$; seleção das unidades de análise ou unidades de significados (orientada pelas questões da pesquisa, incluindo palavras, sentenças, frases, parágrafos ${ }^{33}$ ); análise temática, que pode ser feita a partir de assuntos que ocorrerem com mais frequência ${ }^{34,35}$; e categorização de temas que exprimam significados e elaborações importantes de acordo com os objetivos de estudo. Nessa etapa, a codificação é importante, pois é por meio dela que os dados são organizados em categorias e surgem as características relevantes do conteúdo ${ }^{32}$. A categorização de temas foi feita manualmente, levandose em consideração elementos de representatividade, homogeneidade, pertinência, convergências e divergências.

Depois da indexação dos dados, cada assunto foi analisado e interpretado, utilizando-se como base a revisão da literatura ${ }^{36}$ sobre AA. A fase final de validação foi feita pela triangulação de teorias, na qual se analisam os dados tomando por base várias teorias e tenta-se encontrar validade intrínseca pelo embasamento de cada uma delas ${ }^{37}$.

\section{Aspectos éticos}

O estudo foi aprovado (Parecer no 3.959.822/ Certificado de Apresentação para Apreciação Ética (CAAE) no 21117219.5.0000.5152) pelo Comitê de Ética em Pesquisas em Seres Humanos (CEP) da UFU.

\section{RESULTADOS E DISCUSSÃO}

Inicialmente foi feita uma caracterização dos participantes do GF, seguida da análise aprofundada dos dados produzidos, baseada na literatura sobre SUS, ABS, ESF e AA. Apresentam-se as falas de acordo com as categorizações temáticas, o referencial teórico, as inferências e as interpretações feitas pelas autoras do artigo.

\section{Características dos participantes do grupo focal}

Os participantes são predominantemente do gênero feminino, adultos jovens, com pouca experiência profissional e em ESF. As falas desses indivíduos foram submetidas à análise de conteúdo, sendo geradas as seguintes categorias temáticas: desafios na consolidação da APS e investimento em equipes mínimas; tensionamentos quanto ao aumento na demanda de atendimentos individuais e sobrecarga de trabalho; educação e saúde: intersecções necessárias entre ensino, serviço, gestão e comunidade.

\section{Desafios na consolidação da atenção primária à saúde e investimento em equipes mínimas}

No Cejar, a ESF foi pensada para o acolhimento da residência de $M F C$, que estava sem campo de atuação em APS devido ao fechamento da rede de saúde da prefeitura de Uberlândia, e não propriamente como um desejo da equipe. Os profissionais da UBS tradicional não foram incorporados formalmente à ESF. Os demais também não receberam capacitação teórica nem prática sobre ESF, e nenhum tinha experiência na área. Na verdade, também não houve interesse em capacitação quando novos profissionais vindos da ESF chegaram. Todo o processo ocorreu paralelamente à equipe antiga, com pouco diálogo e interação com a gestão.

Dessa maneira, implementar o AA em uma equipe que não tem ainda identidade com seu modelo de gestão e que não tem profissionais inseridos na comunidade/território prejudicou o funcionamento desse sistema de agendamento. O fato de o Cejar estar em processo de transição de um modelo tradicional para a ESF e ainda não contar com ACS, além de estar falho em relação à territorialização e adscrição de clientela, atrapalhou a implementação do AA, como pode ser verificado nas falas a seguir:

Participante 5: A falta de uma equipe completa, de uma quantidade de funcionários completa [...] acho que isso também complica com um pouco. [...] Eu acho que uns dos grandes problemas que a gente teve durante esse processo é de a gente não ter o cadastramento, o fato da gente não ter uma área descrita, cadastrada, definida, a gente não conhece bem a população.

Participante 2: [...] era impossível o acolhimento que pode ser feito por um ACS, um técnico, podia ser feito por muita gente, ficou concentrado só na enfermagem e isso era um problema, e sem o acolhimento não tem acesso [...].

Para a implementação de APS, são fundamentais ferramentas como territorialização, cadastramento, classificação das áreas de riscos, identificação de vulnerabilidades e estabelecimento de vínculos, elementos que auxiliam a garantia dos direitos à saúde ${ }^{38}$. A territorialização consiste 
em conhecer a área de abrangência da equipe por meio de visitas de campo, identificando desde elementos naturais (relevo, bacia hidrográfica, solo, arborização) até elementos socioeconômicos (casas de alvenaria ou barracos, invasões, abastecimento de energia e água encanada) $)^{39}$.

No Cejar, houve momentos de visita domiciliar de médicos, residentes e enfermeiros que permitiram conhecer o território de maneira parcial. Como o cadastramento da população foi feita por acadêmicos de Medicina, mestrandos da Saúde do Trabalhador e estagiárias de um curso de ACS, pessoas que não participam efetivamente da equipe, o conhecimento das famílias se deu de modo indireto, pouco aprofundado. Não houve discussão após essa coleta de dados, e a classificação de risco das famílias foi iniciada por estudantes de Enfermagem, mas interrompida com a troca frequente de estágios. O processo ficou fragmentado. Implementar o AA é tarefa desafiadora, majoritariamente onde são atendidas grandes quantidades de usuários. Manejar lista de pacientes, readaptar oferta de serviços de saúde e de diferentes categorias profissionais, usar a telemedicina, construir fluxos de atendimento são exemplos do que deve ser repensado, o que exigirá planejamento, sistematização e suporte da gestão ${ }^{25}$. Nas falas a seguir, vê-se que o Cejar não conta com algumas tecnologias que facilitariam a implementação da ESF e do $\mathrm{AA}$, como prontuário eletrônico voltado para a APS (apesar de ter acesso ao prontuário eletrônico hospitalar -Sistema de Informação Hospitalar (SIH) do SUS).

Participante 7: [...] grande problema que a gente tem é que a gente não tem prontuário eletrônico.

Participante 6: É uma coisa que a gente sentiu falta seja para implementar o que for, é uma coisa moderna, é uma falta de instrumento tecnológico instrumento de uso que facilite a nossa vida agenda, prontuário eletrônico, uma agenda eletrônica nossa, isso dificultou e dificulta até hoje o nosso serviço.

Além disso, o trabalho em equipe é elemento fundamental para organização da demanda e dos processos de trabalho. As unidades de saúde poderão melhorar sua capacidade de atendimento se suas enfermeiras e outros profissionais de saúde forem capacitados para lidar com cuidados preventivos e com a rotina de doentes crônicos, sem o envolvimento necessariamente do médico. Algumas clínicas, após adotarem essa "divisão de tarefas", tiveram aumento de sua produtividade e da satisfação da equipe ${ }^{40}$.

A troca e a incorporação de diferentes saberes da interdisciplinaridade promovem a proximidade, comparação, relação e integração dos conhecimentos condizentes com as diversas demandas em saúde atuais ${ }^{41}$. No entanto, a formação dos profissionais de saúde ainda é majoritariamente feita em separado, mesmo que eles tenham no futuro que trabalhar em conjunto ${ }^{42}$.

Participante 6: [...] eu achei que foi muito importante é que a gente começou a trabalhar mais como equipe multidisciplinar [...] a gente ter conhecimento do serviço do outro, que até então fica muito centrado no médico [...] aí junto realmente é uma equipe multi, onde você tem um médico, uma enfermeira, um fisioterapeuta [...].

Participante 2: Criou uma ideia de equipe.

A quinta recomendação do estudo para a APS brasileira mais forte é "ampliar a atuação clínico-assistencial de todas as categorias profissionais das equipes de APS, com a utilização de protocolos multiprofissionais baseados na melhor evidência científica disponível"43 (p.4). Assim, o trabalho colaborativo e multiprofissional retira o foco do cuidado no modelo biomédico e distribui responsabilidades e funções para os diferentes membros da equipe, a qual dialoga entre si e se apoia.

\section{Tensionamentos quanto ao aumento na demanda de atendimentos individuais e sobrecarga de trabalho}

As equipes de APS lidam com dois tipos de demandas, a programada e a espontânea. Com a chegada da residência de MFC no Cejar em 2018, começaram os atendimentos à demanda espontânea:

\begin{abstract}
Participante 7:[...]praticamentenãotinhaatendimento de demanda espontânea, era praticamente só na forma de agendamento, o que tava nos angustiando muito porque o paciente nos procurava com queixa e que às vezes precisava ser resolvido na hora e que a gente não tinha disponibilidade de atendimento [...].
\end{abstract}

Participante 6: Isso é porque, se ela atendia 20, eu e a pessoa 5 atendia 40 , filtrava 20 e atendia 40 , entendeu?

"Certifique-se de que cada médico tenha um tamanho de painel gerenciável, com base em seu escopo de prática clínica, diversidade de pacientes e tempo gasto no consultório"44 (p.60). O painel se refere ao perfil e ao número de pacientes atendidos, e conhecê-lo é fundamental. A Política Nacional de Atenção Básica (Pnab) vigente ${ }^{16}$ recomenda que uma equipe de APS tenha cerca de três mil pessoas em seu painel, número maior que o indicado para realidades socioeconômicas menos desiguais como a dos Estados Unidos, da Inglaterra e de alguns países europeus $^{22}$. Demora por consultas, aumento de insatisfação dos pacientes e prejuízo da longitudinalidade são gerados por painéis excessivamente grandes. O número apropriado de população atendida é a chave para gerenciar carga de trabalho e fornecer atendimento de alta qualidade ${ }^{45}$. 
Se os painéis forem muito grandes, a demanda irá sobrecarregar a capacidade de prestar atendimento, e o acesso rápido será impossível de ser alcançado. Para lidar melhor com o tamanho do painel, as práticas clínicas precisam ser adaptadas, como com consultas por e-mail, atendimentos em grupo e consultas de enfermagem e de outros profissionais de saúde. Como o painel populacional do Cejar ainda é desconhecido, não havia certeza de que realmente a população que estava demandando era da área de abrangência, ainda mais com a falta de ACS. No entanto, isso também mostra como o acesso foi facilitado com o AA: muitas pessoas desconhecidas estavam frequentando a unidade, como mostram os trechos a seguir:

Participante 2: Eu acho que deu acesso a gente que não tinha acesso antes, isso então mudou o perfil de gente que era atendido, veio gente que não vinha [...].

Participante 5: $O$ acesso avançado, ele abre as portas para você ser atendido, ou agendado pelo menos, qualquer dia, qualquer horário, é lógico que pra população foi muito positivo.

\section{Educação e saúde: intersecções necessárias entre ensino, serviço, gestão e comunidade}

O Baylor Family Medicine demonstrou com sua experiência que o agendamento por AA pode ser implementado em um ambiente acadêmico tradicional com benefícios para todos os envolvidos, porém alguns ajustes precisaram ser feitos. As vagas da agenda ao longo da semana variam de acordo com os alunos/preceptores e seus horários (são feitos relatórios diários para confirmar essa disponibilidade), e foi dada ênfase à educação cuidadosa dos pacientes e da equipe quanto ao novo sistema de agendamento adotado. Pesquisas feitas após a implementação mostraram que os pacientes estavam satisfeitos com o AA nesse ambiente de ensino ${ }^{50}$. No Cejar, foram ressaltados alguns pontos relacionados à implementação do AA em uma unidade de ensino:

Participante 6: O Jaraguá é escola, isso não pode se perder, nós temos um posto de PSF, nós trabalhamos com alunos residentes, são alunos, e eles trocam de dois em dois anos, eles têm outras coisas para fazer [...] a gente é uma escola, aprendendo a trabalhar com outro, com a especialidade do outro, o quanto é importante o outro [...].

Participante 7: [...] uma das coisas que estava acontecendo muito no acesso avançado é perder vinculação com o residente [...] é um rodízio muito grande [...] a gente ganha muito quanto a aprendizado, divisão de conhecimento, e a gente perde no sentido de organização do serviço, a gente tem que ter alguma coisa mais sólida.
No Cejar, nem sempre essa horizontalidade do cuidado é possível, visto que os estágios dos graduandos acabam geralmente em um semestre, os residentes só ficam por dois anos na unidade, e os horários deles variam mensalmente. Se a equipe fideliza os pacientes somente a ela mesma, os alunos perdem a oportunidade de aprendizado nos atendimentos, principalmente em se tratando dos residentes, que devem desenvolver autonomia no seu processo de aprendizado. Nem sempre há relatórios detalhados dos alunos que estarão no Cejar divulgados para toda a equipe, no sentido de adaptar o serviço.

Participante 3:Mas não teve um momento de capacitar a equipe, mas não teve um momento de explicar para a população.

Participante 5: [...] a equipe não foi bem treinada $e$ preparada.

Uma das dicas que Murray et al. ${ }^{23}$ (p.47) dão para a implementação do $A A$ é: "implemente o novo sistema mostrando, e não dizendo, aos pacientes como ele funciona. Quando tentamos explicar nossos agendamentos, muitas vezes os tornamos excessivamente complicados". Nos parágrafos anteriores, percebe-se que não há um consenso em relação à necessidade de preparação para implementar o AA (projeto com planejamento, acompanhamento e avaliações), mas a equipe do Cejar sentiu falta de capacitação e apoio. Antes da implementação do $A A$, apenas duas reuniões de equipe foram feitas: uma para apresentação da proposta e consulta da equipe sobre o interesse nesse sistema de agendamento, e a outra após o estudo de demanda, que mostrou que a equipe seria capaz de absorvê-la.

Participante 2: [...] antes era um centro especializado e do nada passa pra um UBS um dia, entra estratégia de saúde da família, seis meses depois acesso avançado, então assim não teve tempo para explicar para população, não sei nem se tinha como falar isso com a população, sabe?, difícil mesmo.

Participante 3: [...] uma pena também é que tudo que a gente mudava a gente causava alguma reação na população que a gente nunca estava preparada, a gente sempre estava um passo atrás.

A primeira etapa para que a participação social ocorra de fato no contexto do SUS é o acesso aos serviços de saúde. A fim de que os brasileiros ajudem na tomada de decisões do sistema de saúde, devem ser feitas campanhas pelos próprios serviços (que influenciam a atuação dos usuários) para que a população seja informada sobre seus direitos e sobre o funcionamento do SUS ${ }^{46}$. Os usuários de saúde devem usufruir do controle social em todos os âmbitos de sua relação com o sistema: na participação de conselhos e conferências; no planejamento, na 
avaliação e na fiscalização de ações em saúde; no conhecimento dos processos de trabalho da sua unidade de referência; no seu contexto comunitário; no seu plano terapêutico; e nas suas necessidades individuais de saúde ${ }^{47}$.

Nas falas a seguir, vê-se que a equipe de saúde e a população apresentaram conflitos importantes na implementação do AA:

Participante 5: Então eu acho que a população não foi orientada, educada, e vem de uma cultura [...] a pessoa quer que seu problema seja resolvido hoje, agora, não importa se o do outro é mais urgente ou não, eu quero resolver o meu [...] eu acho que é possível melhorar a partir do momento que você estabelece as regras, as normas [...].

Participante 6: Os hiperutilizadores estavam sempre aquie era sempreasmesmas pessoas, enós estimulamos que eles poderiam vir em qualquer momento que eles poderiam ser atendidos mesmo [...] a gente aprendeu muito com pandemia a questão de que o paciente não gosta de seguir regras, que o paciente não gosta de ser interpelado, de que o paciente não gosta de normas [...] culturalmente não tem como eu comparar, infelizmente, a população brasileira com a população do Canadá, onde o PSF funciona, não é só dinheiro, gente, é cultura, é a parte social, é muita coisa enraizada [...].

Por mais que um dos pilares do SUS seja a participação social, esta vem sendo permeada historicamente por desafios para a sua execução. A APS deve valorizar e incentivar o controle social, mas ainda vemos muitas relações de poder sendo estabelecidas entre profissionais de saúde e comunidade. A participação dos usuários é necessária para a corresponsabilização na construção de serviços de saúde que efetivamente atendam ao contexto local ${ }^{48}$. Ainda há uma prática dos profissionais latino-americanos de "evangelização sanitária", em que há subordinação da população em ações de educação e participação popular. Os usuários são pouco compreendidos em seu modo de lidar com cuidados em saúde e em seu contexto sociocultural ${ }^{49}$.

A história mostra que, além de princípio do SUS e diretriz da APS brasileira, a participação popular é constituinte primordial das práticas de saúde locais e das comunidades, não como mero discurso, mas como elemento fundamental para a efetivação das ações de saúde. Interesses, pedidos e vontades dos que moravam no território eram acolhidos, apoiados e inseridos nas discussões dos serviços, respondendo aos desafios sociais e promovendo sua qualidade de vida. $A$ participação social, assim, permite que se estabeleça confiança na equipe, as pessoas se sentem acolhidas, com acesso. Trabalhadores que escutam as necessidades e os interesses comunitários, de modo profundo e resolutivo, que consideram e valorizam as pessoas, estabelecem relações de promoção de saúde, de pertencimento, de afetividade. O serviço deve ser construído por meio de diálogo "fraterno e conflituoso" entre os saberes científicos e os saberes populares, acrescentando a eles a integralidade. Nenhum conhecimento ou ideia se sobreporá, mas a união, na verdade um novo saber, produto do diálogo de ideias diversas, gerará melhores decisões em saúde para o contexto local ${ }^{50}$.

O presente estudoficou limitado metodologicamente em relação à coleta de dados. Talvez a percepção dos profissionais em relação ao AA no Cejar ficaria mais completa se entrevistas permitissem conhecer opiniões não generalizáveis, e se tivesse sido realizada a observação participante com diários de campo durante a implementação do sistema de agendamento em questão. Como o GF ocorreu a posteriori e durante a pandemia da coronavirus disease 2019 (Covid-19), também existe o viés de memória dos participantes. Outras limitações: imprevisibilidade do moderador durante a condução do GF, possível inibição dos participantes devido ao local do GF (no próprio ambiente de trabalho) e impossibilidade de generalizações de processos para outras realidades, já que foi analisada uma única realidade. Como contribuição, foi suscitada a discussão sobre o acesso aos serviços de saúde por meio da agenda das equipes de APS. Levantaram-se alguns desafios do AA em uma unidade acadêmica de saúde, em que a ESF não está consolidada, e acerca da sobrecarga da equipe, pontos pouco trabalhados na literatura atual sobre o tema.

\section{CONSIDERAÇÕES FINAIS}

O acesso aos serviços de saúde ainda é um desafio para o SUS, por mais que a APS e a ESF o tenham ampliado nas duas últimas décadas no Brasil. Vários aspectos permeiam o acesso dos usuários, desde linguagem, humanização, localização geográfica e até agenda. O AA é uma proposta moderna de agendamento de consultas médicas que deixa o serviço livre a maior parte do tempo para as pessoas que chegam no mesmo momento.

No Cejar, muitas questões influenciaram implementação do AA: não ter equipe mínima de ESF (financiamento insuficiente), com destaque para a falta do serviço do ACS na comunidade, territorialização e conhecimento da população adscrita frágeis, painel populacional supostamente inadequado com consequente sobrecarga de trabalho, quase nenhuma capacitação, pouco envolvimento da gestão, falta de ferramentas digitais, horizontalidade do cuidado prejudicado (principalmente por se tratar de uma unidade acadêmica de saúde), não envolvimento da população em relação à escolha desse tipo de agendamento e falta de instrumentos dialógicos para lidar com os usuários. Contudo, muitos pacientes, antes desconhecidos, tiveram a oportunidade de frequentar o serviço, o trabalho passou a ser mais colaborativo por parte da equipe multiprofissional, e a 
pandemia da Covid-19 de certa forma favoreceu o aprendizado sobre o AA, no sentido de ampliar a resolutividade dos indivíduos que procuravam o serviço em uma só ocasião ou de facilitar o acesso por outros meios, como por teleconsultas.

Conclui-se que a experiência do Cejar apresentou particularidades durante a implementação do $\mathrm{AA}$. $\mathrm{O}$ acesso foi certamente facilitado, porém às custas de um desgaste grande dos trabalhadores, e várias fragilidades do serviço vieram à tona. Estratégias que melhorem o acesso sempre devem ser levantadas, mas elementos básicos que dão suporte a essas estratégias devem ser prioridade, como equipes completas, adscrição de clientela, prontuário eletrônico, envolvimento da gestão nos processos de trabalho da unidade e participação social. Outras equipes que também queiram implementar o $A A$ podem usar os nós críticos aqui apresentados para um melhor planejamento dessa agenda. Mais estudos sobre a experiência da implementação do AA em contextos diversos devem ser encorajados para que políticas públicas que abordem o acesso do usuário ao sistema de saúde sejam desenvolvidas.

\section{CONTRIBUIÇÃO DAS AUTORAS}

As autoras colaboraram igualmente na elaboração deste estudo.

\section{CONFLITO DE INTERESSES}

Declaramos não haver conflito de interesses.

\section{FINANCIAMENTO}

Declaramos não haver financiamento.

\section{REFERÊNCIAS}

1. Starfield B. Atenção primária: equilíbrio entre a necessidade de saúde, serviços e tecnologias. Brasília: Unesco, Ministério da Saúde; 2002.

2. Norman $\mathrm{AH}$, Tesser CD. Acesso ao cuidado na Estratégia Saúde da Família: equilíbrio entre demanda espontânea e prevenção/promoção da saúde. Saúde Soc. 2015;24(1):165-79

3. Tesser CD, Norman AH. Repensando o acesso ao cuidado na Estratégia Saúde da Família. Saúde Soc. 2014;23(3):869-83.

4. Silva LA, Casotti CA, Chaves SCL. A produção científica brasileira sobre a Estratégia Saúde da Família e a mudança no modelo de atenção. Cien Saude Colet. 2013;18(1): 221-32

5. de Sousa MF. O Programa Saúde da Família no Brasil: análise do acesso à atenção básica. Rev Bras Enferm. 2008;61(2):153-8.

6. Teixeira CF, Solla JP. Modelo de atenção à saúde no SUS: trajetória do debate conceitual, situação atual, desafios e perspectivas. In: Lima NT, Gerschman S, Edler FC. Saúde e democracia: história e perspectivas do SUS. Rio de Janeiro: Fiocruz; 2005. p. 451-79.

7. de Azevedo ALM, Costa AM. A estreita porta de entrada do Sistema Único de Saúde (SUS): uma avaliação do acesso na Estratégia de Saúde da Família. Interface Comun Saúde Educ. 2010;14(35):797-810.

8. Barbosa SP. Acesso à atenção primária à saúde em municípios de uma região de saúde de Minas Gerais [dissertação]. Belo Horizonte: Universidade Federal de Minas Gerais; 2013 [acesso em $1^{\circ}$ nov 2019]. Disponível em: http://www.cienciaesaudecoletiva.com.br/artigos/oacesso-a-partir-da-atencao-primaria-a-saude-em-tres-municipios-doestado-de-minas-gerais-a-otica-dos-profissionais-de-saude/11858.
9. Luz J. Implantação do processo de trabalho da Estratégia Saúde da Família em Florianópolis: uma exploração quali-quantitativa [tese]. Florianópolis: Universidade Federal de Santa Catarina; 2009 [acesso em 10 out 2020]. Disponível em: https://repositorio.ufsc.br/bitstream/ handle/123456789/120115/282529.pdf?sequence $=1$.

10. Silva SA, Baitelo TC, Fracoli LA. Avaliação da atenção primária à saúde: a visão de usuários e profissionais sobre a Estratégia de Saúde da Família. Rev Lat Am Enfermagem. 2015;23(5):979-87.

11. de Paula WKAS, Samico IC, Caminha MFC, da Silva SL. Primary health care assessment from the users' perspectives: a systematic review. Rev Esc Enferm USP. 2016;50(2):335-45.

12. Marin MJS, Marchioli M, Dutra MYA. Fortalezas e fragilidades do atendimento nas unidades básicas de saúde tradicionais e da Estratégia de Saúde da Família pela ótica dos usuários. Texto \& Contexto Enferm. 2013;22(3):780-8.

13. Sisson $M C$, de Andrade $S R$, Giovanella $L$, de Almeida PF, Fausto $M C R$, de Souza CRP. Estratégia de Saúde da Família em Florianópolis: integração, coordenação e posição na rede assistencial. Saúde Soc. 2011;20(4):991-1004.

14. van Stralen CJ, Belisário SA, van Stralen TBS, de Lima AMD, Massote AW, Oliveira CL. Percepção dos usuários e profissionais de saúde sobre atenção básica: comparação entre unidades com e sem saúde da família na Região Centro-Oeste do Brasil. Cad Saude Publica. 2008;24(supl 1):s148-58.

15. Conill EM. Ensaio histórico-conceitual sobre a atenção primária à saúde: desafios para a organização de serviços básicos e da Estratégia Saúde da Família em centros urbanos no Brasil. Cad Saude Publica. 2008;24(supl 1):s7-16.

16. Brasil. Portaria $n^{\circ} 2.436$, de 21 de setembro de 2017. Aprova a Política Nacional de Atenção Básica, estabelecendo a revisão de diretrizes para a organização da Atenção Básica, no âmbito do Sistema Único de Saúde (SUS). Brasília: Ministério da Saúde; 2017 [acesso em $1^{\circ}$ out 2020]. Disponível em: https://bvsms.saude.gov.br/bvs/saudelegis/gm/2017/ prt2436_22_09_2017.html.

17. Campos RTO, Ferrer AL, da Gama CAP, Campos GWS, Trapé TL, Dantas DV. Avaliação da qualidade do acesso na atenção primária de uma grande cidade brasileira na perspectiva dos usuários. Saúde Debate. 2014;(38 supl esp):252-64.

18. Ryu J, Lee TH. The waiting game: why providers may fail to reduce wait times. N Engl J Med. 2017;376(24):2309-11.

19. Knight A, Lembkel T. Appointments 101: how to shape a more effective appointment system. Aust Fam Physician. 2013;42(3):152-6.

20. Murray M, Berwick DM. Advanced access: reducing waiting and delays in primary care. JAMA. 2003;289(8):1035-40.

21. Murray M, Tantau C. Redefining open access to primary care. Manag Care Q. 1999;7(3):45-55.

22. Vidal TB, Rocha SA, Harzheim E, Hauser L, Tesser CL. Modelos de agendamento e qualidade da atenção primária: estudo transversal multinível. Rev Saude Publica. 2019; 53(38):1-10.

23. Murray M, Tantau C. Same-day appointments: exploding the access paradigm. Fam Pract Manag. 2000;7(8):45-50.

24. Brasil. Política Nacional de Humanização da Saúde: gestão participativa e cogestão. Brasília: Ministério da Saúde; 2009.

25. Pereira NVS. A implantação do acesso avançado no serviço de atenção primária do Hospital de Clínicas de Porto Alegre [tese]. Porto Alegre: Universidade Federal do Rio Grande do Sul, 2018 [acesso em $1^{\circ}$ out 2019]

26. Cardoso FM, Campos GWS. Aprendendo a clínica do sofrimento social: narrativas do internato na atenção primária à Saúde. Cien Saude Colet 2020;25(4):1251-60.

27. Coelho BP, Miranda GMD, Coutinho Neto OB. A formação-intervenção na atenção primária: uma aposta pedagógica na educação médica. Rev Bras Educ Med. 2019;43(1 supl 1):632-40.

28. Instituto Brasileiro de Geografia e Estatística. Censo 2010 [acesso em 22 dez 2020]. Disponível em: https://censo2010.ibge.gov.br/.

29. Trad LAB. Grupos focais: conceitos, procedimentos e reflexões baseadas em experiências com o uso da técnica em pesquisas de saúde. Physis. 


\section{9;19(3):777-96.}

30. Bardin L. Análise de conteúdo. Lisboa: Edição 70; 1977.

31. Rodrigues MSP, Leopardi MT. O método de análise de conteúdo: uma versão para enfermeiros. Fortaleza: Fundação Cearense de Pesquisa e Cultura; 1999.

32. Franco MLPB. O que é análise de conteúdo. São Paulo: Cadernos de Psicologia da Educação; 1986. p. 1-31.

33. Downe-Wamboldt B. Content analysis: method, applications, and issues. Health Care Women Int. 1992;13(3):313-21.

34. Kitzinger J, Farquhar C. The analytical potential of "sensitive moments" in focus group discussions. In: Barbour RS, Kitzinger J, editors. Developing focus group research. London: Sage; 1999. p. 156-72.

35. Minayo MCS, Souza, C. O desafio do conhecimento: pesquisa qualitativa em saúde. São Paulo: Hucitec; 2004. p. 201-19.

36. Ressel LB, Beck CLC, Gualda DMR, Hoffmann IC, Silva RM, Sehnem GD. O uso do grupo focal em pesquisa qualitativa. Texto \& Contexto Enferm. 2008; 17 (4): 779-786.

37. Campos CJG. Método de análise de conteúdo: ferramenta para a análise de dados qualitativos no campo da saúde. Rev Bras Enferm. 2004;57(2):611-4.

38. Araújo GB, Alves Filho FWP, Santos RS, Lira RCM. Territorialização em saúde como instrumento de formação para estudantes de medicina: relato de experiência. Sanare. 2017;16(01):124-9.

39. Souza GJ, Gomes C, Zanetti VR. Estratégia da Saúde da Família: a dimensão articuladora do território. Barbarói. 2020;56:141-63.

40. Ghorob A, Bodenheimer T. Three building blocks for improving access to care. Fam Pract Manag. 2013;20(5):12-7.

41. de Oliveira LC, Ávila MMM, Gomes AMA, Sampaio MHLM. Participação popular nas ações de educação em saúde: desafios para os profissionais da atenção primária. Interface. 2014;18(supl 2):1389-400.

42. da Costa MV. A educação interprofissional no contexto brasileiro: algumas reflexões. Interface Comun Saúde Educ. 2016;20(56):197-98.

43. Tasca R, Massuda A, CarvaloWM, Buchweitz C, Harzheim E. Recomendações para o fortalecimento da atenção primária à saúde no Brasil. Rev Panam Salud Pública. 2020;44:e4.

44. Steinbauer JR, Korell K, Erdin J, Spann SJ. Implementing open-access scheduling in an academic practice. Fam Pract Manag. 2006;13(3):59-64.

45. Murray $M$, Davies $M$, Boushon $B$. Panel size: how many patients can one doctor manage? Fam Pract Manag. 2007;14(4):44-51.

46. Vázquez ML, da Silva MRF, Campos ES, de Arruda IKG, Diniz AS, Veras IL, et al. Participação social nos serviços de saúde: concepções dos usuários e líderes comunitários em dois municípios do Nordeste do Brasil. Cad Saude Publica. 2003;19(2):579-91.

47. Cunha JPP, Cunha RE. Sistema Único de Saúde: princípios. In: Ministério da Saúde. Gestão municipal de saúde: textos básicos. Rio de Janeiro: Ministério da Saúde; 2001 [acesso em 22 dez 2020] Disponível em: http:// bvsms.saude.gov.br/bvs/publicacoes/0103gestao1.pdf.

48. Camargo-Borges C, Mishima SM. A responsabilidade relacional como ferramenta útil para a participação comunitária na atenção básica. Saúde Soc. 2009;18(1):29-41.

49. Keijzer B. Los discursos de la educación y participación en salud: de la evangelización sanitaria al empoderamiento. Rio de Janeiro: Fiocruz; 2005.

50. Brutscher VJ, Cruz PJSC. Participação social na perspectiva da educação popular: suas especificidades e potencialidades na atenção primária à saúde. Cadernos CIMEAC. 2020;10(1):126-52. 